\title{
EDITORIAL
}

\section{Data on women in physics}

\author{
Women remain under-represented in science, and in particular in physics. Effective action to \\ remedy this situation should be based on rigorous analysis of high-quality data.
}

In April, the Australian Minister for Industry, Science and Technology, Karen Andrews, launched a 10-year plan to increase the number of women in STEM (science, technology, engineering and mathematics) in Australia ${ }^{1}$. One of the six planks of the plan is evaluation, in other words, to collect data to measure how women-in-STEM schemes actually perform, to decide which schemes should be supported further.

Of course, the under-representation of women in science, and in particular in physics, is profound enough that we don't need detailed statistics to see its existence. Additionally, each woman who has had a career in science is a unique individual whose personal experiences are worth listening to. However, with inadequate data collection and analysis, progress towards equal representation of women remains slow. Indeed, discussions continue $^{2}$ about what's best to do: attract girls in school; improve working conditions for female mid-career scientists; or something else?

Faced with this discussion, we wanted to better grasp the situation in physics. Doing so requires going beyond statistics that are aggregated for all of the STEM subjects, only consider a certain career stage or only concern a particular country. Therefore, along with Nature Reviews Chemistry, we decided to explore the proportion of men and women in physics and chemistry, respectively, around the world.

The first thing that stands out from these reports is the difference between two physical science fields that are in many ways close cousins. In physics, the intake of undergraduate students is typically $20 \%$ female in countries such as the USA or UK. In chemistry, this fraction is double, at around $40 \%$. This difference is not visible in aggregated data for STEM, but explaining it could provide insights that would be useful to both communities of scientists.

It is also clear that not all countries collect highquality data, and of those that do, not all collect data on the same career stages. Different countries have different policies and cultures, and meaningful international comparisons might make it possible to disentangle some of the factors leading to success or failure in recruiting and retaining female physicists.
When looking at recruitment of girls to physics classes, one alternative to large-scale international comparisons is small-scale controlled studies taking place in schools and universities. For example, this year the Institute of Physics in the UK invited secondary schools to take part in a randomized controlled trial of interventions aimed at encouraging girls to study physics ${ }^{3}$. However, it's impossible to do controlled studies on peoples' careers, so international comparisons could play an important role in guiding actions aimed at retaining women after graduation.

Besides data collection, there is also a place for innovative analyses of existing data. In this issue, we have a Comment from Jichao Li and colleagues, who have done a statistical analysis of a data set on almost all Nobel Prize winners in physics, chemistry, and physiology or medicine, looking for trends in the time of publication and team size of Nobel Prize-winning work. Applying this type of analysis to the data on women in physics could help select between hypotheses about what drives the directions of women's careers.

Wanting comprehensive data and quantitative analysis is not the same thing as advocating waiting to take action. Women leaving physics research - or never entering it to begin with - is a tremendous waste of talent and it is clear that many women who could enjoy a career in physics are being denied this opportunity. The problem is serious and it is appropriate to take action wherever possible.

We believe that action should be informed by rigorous analysis of solid data. To this end, we would like to see international coordinated efforts to standardize the collection of demographic data, so that comparisons can be made. We would also like to see more in-depth statistical analyses that make it possible to decide between possibilities when making interventions.

1. Australian Academy of Science. Women in science, technology, engineering and mathematics (STEM) decadal plan. Australian Academy of Science http://www.science.org.au/womeninSTEMplan (2019).

2. Jamieson, V. Women in physics: why there's a problem and how we can solve it. New Scientist https://www. newscientist.com/article/ mg24032031-900-women-in-physics-why-theres-a-problemand-how-we-can-solve-it/ (2018).

3. Henshaw, P. Physics gender balance trial. Sec Ed http://www.sec-ed co.uk/resources-products/physics-gender-balance-trial/ (2019). 